\title{
Biological properties of lipopolysaccharides from Bordetella species
}

\author{
Mitsuru Watanabe, ${ }^{1}$ Hiroaki Takimoto, ${ }^{1}$ Yoshio Kumazawa ${ }^{2}$ and Ken-IChi Amano ${ }^{3 *} \dagger$ \\ ${ }^{1}$ The Kitasato Institute and ${ }^{2}$ School of Pharmaceutical Sciences, Kitasato University, 5-9-1 Shirokane, Minato-ku, \\ Tokyo 108, Japan \\ ${ }^{3}$ Department of Bacteriology, Hirosaki University School of Medicine, Hirosaki 036, Japan
}

(Received 25 July 1989; revised 30 October 1989; accepted 28 November 1989)

\begin{abstract}
Biological activities of lipopolysaccharides (LPS) extracted from Bordetella pertussis, B. parapertussis and $B$. bronchiseptica were compared with those of Escherichia coli LPS. The LPS preparations from $B$. pertussis showed biological activities comparable to those of $E$. coli LPS in terms of lethal toxicity in galactosamine-sensitized mice, pyrogenicity in rabbits, mitogenicity in $\mathrm{C} 3 \mathrm{H} / \mathrm{He}$ spleen cell cultures, macrophage activation, and induction of tumour necrosis factor. All the activities of LPS preparations from $B$. parapertussis, except mitogenicity, were lower than those of $E$. coli LPS. LPS from $B$. parapertussis gave the greatest mitogenic action of all those tested. Biological activities stronger than or comparable to those of $E$. coli LPS were observed for LPS from $B$. bronchiseptica.
\end{abstract}

\section{Introduction}

Lipopolysaccharides (LPS) extracted from Gram-negative bacteria are potent molecules which elicit in animals a range of characteristic responses. These are often due to stimulation of host mediators such as interleukin-1 and tumour necrosis factor (TNF) (Haeffner-Cavaillon et al., 1984; Nowotny, 1985). Among these responses are the induction of the Shwartzman reaction (Movat \& Burrowes, 1985), antitumour effects (Freinman et al., 1987) and adjuvant activity (McGhee et al., 1980).

LPS from Bordetella pertussis also exhibits the biological activities described above (Chaby \& Caroff, 1988), although lipid A derived from this LPS is non-toxic and does not induce the Shwartzman reaction (Ayme et al., 1980). There is little information on the biochemical, immunological and biological properties of LPS from the other two species of Bordetella, namely $B$. parapertussis and $B$. bronchiseptica. In the preceding paper (Amano et al., 1990) we described the biochemical and immunological properties of preparations of LPS from the three species of Bordetella, and in the present paper we give some of their biological activities, i.e. lethal toxicity in

† Present address: Central Research Laboratory, Akita University School of Medicine, Akita 010, Japan.

Abbreviations: LPS, lipopolysaccharide(s); TNF, tumour necrosis factor: i.v., intravenously; i.p., intraperitoneal(ly); LAL, limulus amoebocyte lysate. galactosamine-sensitized mice, pyrogenicity, mitogenicity, macrophage activation and TNF-inducing activity. These activities of Bordetella LPS were compared to those of Escherichia coli LPS.

\section{Methods}

Organisms and extraction of LPS fractions. B. pertussis strains Tohama [phase I, BpI(тоHAMA)] and AK-168 [phase I, BpI(AK-168)], B. parapertussis strains 21815 [Bpp(21815)] and AK-167 [Bpp(AK167)], and B. bronchiseptica strains $\mathrm{L} 3[\mathrm{Bbs}(\mathrm{L} 3)]$ and $\mathrm{H}-214$ [Bbs(H214)] were obtained from our laboratory stock. Cultivation and purification of Bordetella strains, extraction of LPS with hot phenol/water, and purification by ultracentrifugation and by treatment with proteolytic enzymes were carried out as described previously (Amano et al., 1990). LPS from E. coli O55:B5 was purchased from Difco.

Determination of lethal toxicity. This was done by the method described by Galanos et al. (1979). Various concentrations of LPS in $0.2 \mathrm{ml}$ water were injected intravenously (i.v.) into male 10 -week-old C57B1/6 mice (Shizuoka Animal Center, Shizuoka, Japan) immediately after intraperitoneal (i.p.) administration in $0.5 \mathrm{ml}$ of pyrogen-free saline containing $10 \mathrm{mg}$ D-galactosamine (Sigma).

Determination of pyrogenicity. Pyrogenicity was determined in Japanese white rabbits (2.1-2.4 kg) (Gokita Breeding Service, Tokyo, Japan) of the same sex in groups of three for each dose to be tested, as described by Kumazawa et al. (1988). The sample was injected i.v. into three rabbits. Rectal temperature was monitored continuously for $3 \mathrm{~h}$, and the maximal increase in temperature was recorded for each rabbit.

Measurement of limulus amoebocyte lysate (LAL) gelation. LAL gelation activity was tested using Pregel-S reagent (Seikagaku Kogyo Co.). 


\begin{abstract}
Measurement of macrophage activation. This was done by measuring phagocytic, cellular lysosomal enzyme (acid phosphatase), and cytostatic activities of macrophages obtained from the peritoneal cavity of ICR mice (Shizuoka Animal Center) which had been injected i.p. with $1 \mu \mathrm{g}$ of test sample $4 \mathrm{~d}$ earlier, as described by Kumazawa et al. (1988).

Phagocytic activity was assessed by measuring the radioactivity of ${ }^{51}$ Cr-labelled, antibody-sensitized sheep erythrocytes which were phagocytosed by in vivo-stimulated peritoneal macrophages. Acid phosphatase activity was measured as follows. Peritoneal macrophages stimulated in vivo were lysed in 96-well microplates with $0.1 \%$ Triton X100 , and $20 \mu \mathrm{l} 0.2 \mathrm{M}$-sodium acetate buffer (pH 5.0) plus $20 \mu \mathrm{l} 24 \mathrm{mM}-p$ nitrophenyl phosphate were added to the macrophage lysates. After incubation at $37^{\circ} \mathrm{C}$ for $30 \mathrm{~min}, 200 \mu \mathrm{l} 0.2 \mathrm{M}$ sodium carbonate was added to the reaction mixture, and the $A_{405}$ was measured. Enzyme activity was calculated from a standard curve made using known amounts of potato acid phosphatase. Cytostasis-inducing activity was assessed by measuring the growth-inhibitory action against EL-4 lymphoma cells. The activity was calculated as the percentage inhibition of $\left[{ }^{3} \mathrm{H}\right]$ thymidine incorporation into EL-4 cells.
\end{abstract}

Assay of TNF-inducing activity. This was done by the method described by Kumazawa et al. (1988). Briefly, $10 \mu \mathrm{g}$ LPS was administered i.v. into ICR mice (Charles River Japan, Atsugi, Japan) primed with Propionibacterium acnes. Sera were separated from blood $1.5 \mathrm{~h}$ after administration of LPS and treated at $56^{\circ} \mathrm{C}$ for $30 \mathrm{~min}$ before use. The TNF activity in the sera was determined by measuring the percentage inhibition of $\left[{ }^{3} \mathrm{H}\right]$ thymidine uptake into $\mathrm{L} 929$ cells.

Determination of mitogenic activity. Spleen cells, obtained from $\mathrm{C} 3 \mathrm{H} / \mathrm{He}$ and $\mathrm{C} 3 \mathrm{H} / \mathrm{HeJ}$ mice (Charles River Japan), were suspended in RPMI 1640 medium supplemented with $10 \%(v / v)$ foetal calf serum, $25 \mathrm{mM}$-HEPES, $2 \mathrm{~mm}$-L-glutamine, 100 units penicillin $\mathrm{ml}^{-1}$ and $100 \mu \mathrm{g}$ streptomycin $\mathrm{ml}^{-1}$, and incubated in triplicate at $37^{\circ} \mathrm{C}$ for $48 \mathrm{~h}$ in the presence or absence of LPS preparations (Kumazawa et al., 1988). The cells were given $\left[{ }^{3} \mathrm{H}\right]$ thymidine $4 \mathrm{~h}$ before harvest, and mitogenicity was assayed by determining the radioactivity incorporated into the cells.

\section{Results}

\section{Toxicity, pyrogenicity and $L A L$ gelation activity}

Toxicity in galactosamine-sensitized mice is shown in Table 1. Among the preparations of LPS tested, Bbs(L3) was the most toxic, while Bbs(H-214) LPS and two samples of $B$. pertussis LPS possessed toxicity similar to that of $E$. coli LPS. Weak toxic activity was shown for two preparations from $B$. parapertussis.

The smallest dose of LPS that consistently evoked a rise in body temperature (over $0.6{ }^{\circ} \mathrm{C}$ per rabbit) was $1 \mathrm{ng} \mathrm{kg}^{-1}$ for $\mathrm{Bbs}(\mathrm{L} 3)$, and $10 \mathrm{ng} \mathrm{kg}^{-1}$ for BpI(TOHAMA), Bbs(H-214) and E. coli LPS; for BpI(AK-168), $\mathrm{Bpp}(21815)$ and $\mathrm{Bpp}(\mathrm{AK}-167)$ a dose of $100 \mathrm{ng} \mathrm{kg}^{-1}$ was required to induce a fever response (Table 1).

In the LAL gelation test, all the LPS preparations from Bordetella species showed activity the same as [BpI(AK168), Bpp(21815), Bpp(AK-167) and Bbs(H-214)] or stronger than [BpI(TOHAMA) and Bbs(L3)] the LPS from E. coli (Table 1).

\section{Macrophage activation}

This was determined by measuring phagocytosis of ${ }^{51} \mathrm{Cr}-$ labelled antibody-sensitized sheep erythrocytes (Fig. 1a), increased level of cellular lysosomal enzyme (acid phosphatase) (Fig. 1b), and induction of cytostasis (Fig. 1c). All the LPS preparations from Bordetella species induced significant macrophage activation at a dose of $1 \mu \mathrm{g}$ per mouse. At this dose level, the most active LPS was Bbs(L3), which had an activity comparable to that of $E$. coli LPS. The two LPS preparations from B. pertussis and that from $\mathrm{Bbs}(\mathrm{H}-214)$ were weaker than the LPS from $E$. coli, and the activities of the two preparations from $B$. parapertussis were low.

\section{TNF-inducing activity}

The two preparations of LPS from $B$. pertussis and the two from $B$. bronchiseptica exhibited TNF-inducing activity comparable to that of LPS from E. coli (Fig. 2). However, the two preparations from $B$. parapertussis were less active than LPS from $E$. coli.

\section{Mitogenic activity}

The LPS preparations from BpI(TOHAMA), two strains of $B$. parapertussis and $\mathrm{Bbs}(\mathrm{H}-214)$ showed much higher mitogenic activity than $E$. coli $\mathrm{LPS}$ in $\mathrm{C} 3 \mathrm{H} / \mathrm{He}$ spleen cell cultures at a concentration of $10 \mathrm{ng}$ per $0.1 \mathrm{ml}$ per well (Fig. 3). The mitogenic activity of LPS from BpI(AK$168)$ and $\mathrm{Bbs}(\mathrm{L} 3)$ was comparable to that of LPS from $E$. coli. None of the LPS preparations from Bordetella species showed significant activity in $\mathrm{C} 3 \mathrm{H} / \mathrm{HeJ}$ mice at the same concentration as used in $\mathrm{C} 3 \mathrm{H} / \mathrm{He}$ mice (data not shown).

\section{Discussion}

LPS from B. pertussis is known to have activities similar to those of the LPS from Gram-negative enterobacteria. The endotoxic activities of LPS, such as fever, Schwartzman reactivity and depression of blood pressure leading to shock and death, are produced by the endotoxin from B. pertussis (Finger et al., 1976; Ayme et al., 1980). Our data demonstrate the toxicity of LPS from other Bordetella species. The LPS preparations from $B$. brochiseptica were similar in toxicity to LPS from $B$. pertussis and $E$. coli, whereas LPS from $B$. parapertussis had lower toxicity.

Amano et al. (1990) showed molecular mass differences of LPS from three species of Bordetella by SDSPAGE: LPS from $B$. pertussis contained two low molecular mass bands, LPS from $B$. parapertussis 


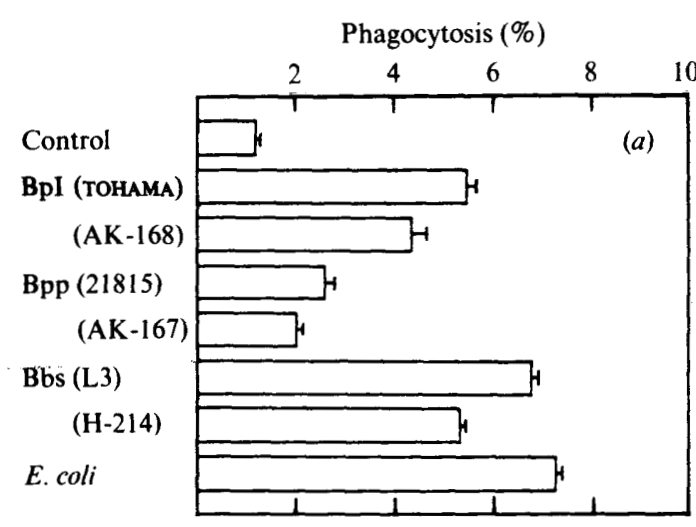

Acid phosphatase [mU (mg protein) $\left.)^{-1}\right]$

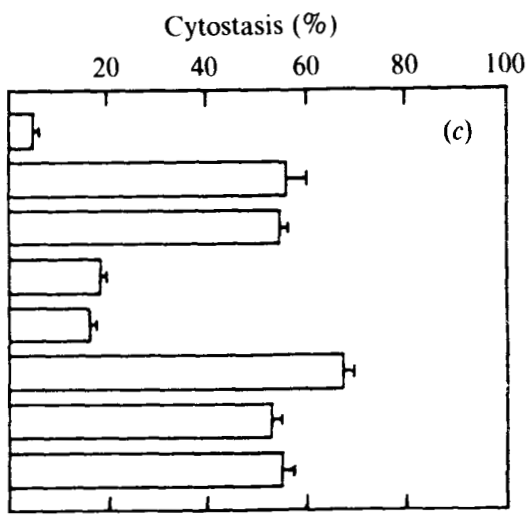

Fig. 1. Potency of peritoneal macrophages activated in vivo with LPS from Bordetella species or E. coli. Peritoneal macrophages were obtained from ICR mice injected i.p. with $1 \mu \mathrm{g}$ LPS $4 \mathrm{~d}$ before. Control experiments were done with macrophages from unstimulated mice. The results are means \pm standard deviation of three separate experiments. (a) Phagocytic activity measured using ${ }^{51} \mathrm{Cr}-\mathrm{labelled}$, antibody-sensitized sheep erythrocytes. (b) Acid phosphatase activity of lysates of $1 \times 10^{5}$ cells determined as $A_{405}$ using $p$-nitrophenyl phosphate as substrate. (c) Cytostatic activity measured using macrophages co-cultivated for $40 \mathrm{~h}$ with target EL-4 lymphoma cells in an effector: target cell ratio of $10: 1$. The activity was assessed by the degree of inhibition of $\left[{ }^{3} \mathrm{H}\right]$ thymidine uptake into the target cells.

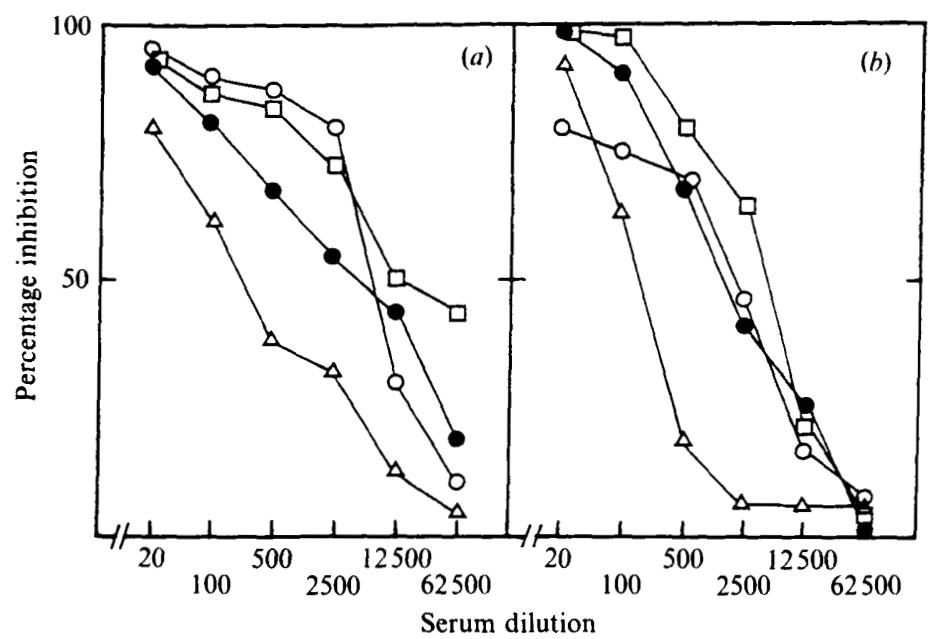

Fig. 2. TNF-inducing activity of LPS from Bordetella species or E. coli. LPS was administered i.v. at a dose of $1 \mu \mathrm{g}$ into ICR mice primed with Propionibacterium acnes. Sera were obtained $1.5 \mathrm{~h}$ after administration of LPS. TNF activity was expressed as the percentage inhibition of $\left[{ }^{3} \mathrm{H}\right]$ thymidine uptake into L929 cells. (a) O, BpI(TOHAMA) LPS; $\triangle$, Bpp(21815) LPS; $\square$, Bbs(L3) LPS;,$E$. coli LPS. (b) O, BpI(AK-168) LPS; $\triangle$, Bpp(AK-167) LPS; $\square$, Bbs(H-214) LPS;,$E$. coli LPS. The results are from a single experiment.

contained ladder structural bands in the high molecular mass area and a single low molecular mass band, and LPS from $B$. bronchiseptica contained ladder structural high molecular mass bands and two low molecular mass bands. Peppler (1984) and Li et al. (1988) also demonstrated the presence of two different LPS components in $B$. pertussis, by SDS-PAGE and immunoblotting. These physical differences could be reflected in the difference of the toxicity among Bordetella LPS.

Lehmann et al. (1987) reported that TNF may be a mediator of toxicity of LPS from Gram-negative bacteria in galactosamine-sensitized mice. Our data, showing that the TNF-inducing activity of Bordetella LPS correlates with toxicity, support this idea. The macrophage activation ability of the various preparations of Bordetella LPS also correlated with their toxicity. Lasfargues et al. (1986) also reported that $B$. pertussis endotoxin is able to induce in mouse peritoneal macrophages cytostatic activity against tumour cells, and that this activity was due to the lipid region of the endotoxin.

Endotoxins from $B$. pertussis are known to be potent mitogens and polyclonal activators for murine B lymphocytes (Girard et al., 1981; Haeffner-Cavaillon et al., 1982). Girard et al. (1981) reported that $B$. pertussis LPS induces a mitogenic response in $\mathrm{C} 3 \mathrm{H} / \mathrm{HeJ} \mathrm{B}$ lymphocytes (which are unresponsive to enterbacterial LPS) as 


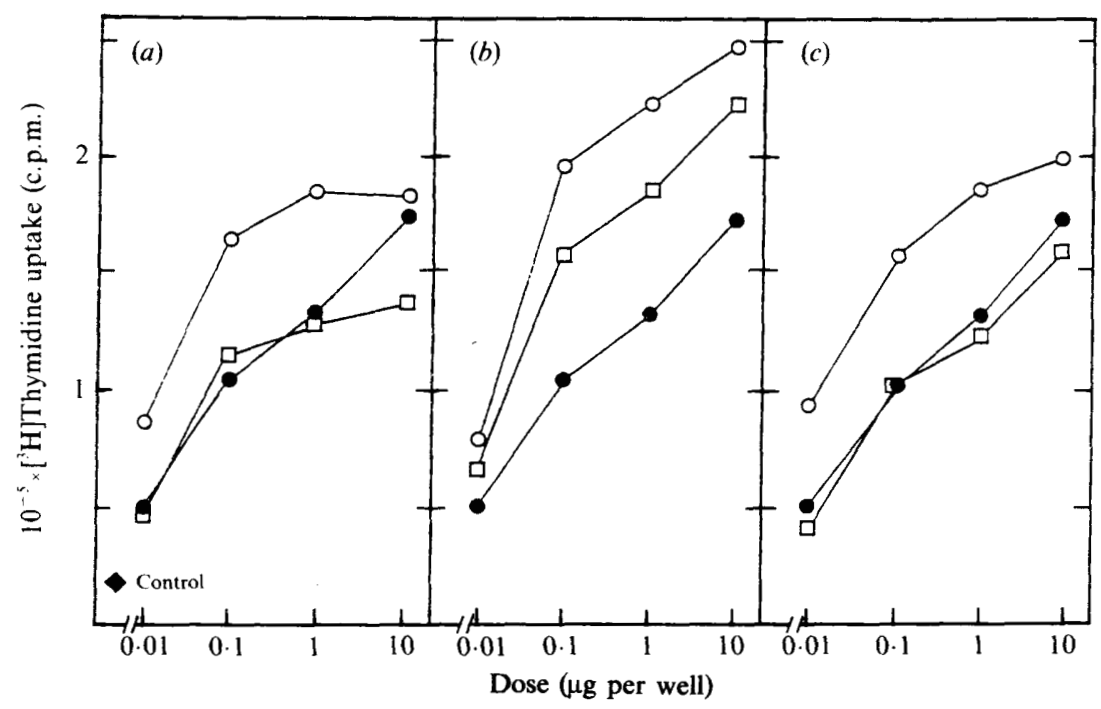

Fig. 3. Mitogenic activity of LPS from Bordetella species or $E$. coli. C3H/He spleen cells were stimulated for $48 \mathrm{~h}$ with the indicated dose of LPS. Mitogenic activity is expressed as mean $\left[{ }^{3} \mathrm{H}\right]$ thymidine uptake of triplicate cultures. (a) $\bigcirc$, BpI(TOHAMA) LPS; $\square, \mathrm{BpI(AK-168)}$ LPS; , E. coli LPS. (b) O, Bpp(21815) LPS; $\square$, Bpp(AK-167) LPS; , E. coli LPS. (c) O, Bbs(H-214) LPS; $\square$, Bbs(L3) LPS;,$E$. coli LPS.

Table 1. Endotoxic activities of LPS preparations from Bordetella species and $E$. coli

\begin{tabular}{lccc}
\hline LPS & $\begin{array}{c}\text { Lethal toxicity } \\
\text { MLD (ng) }\end{array}$ & $\begin{array}{c}\text { LAL gelation } \\
\text { activity (ng ml }\end{array}$ & $\begin{array}{c}\text { Pyrogenicity } \\
\left.\text { MED (ng kg })^{-1}\right)\end{array}$ \\
\hline BpI(TOHAMA) & 1 & $0 \cdot 01$ & 10 \\
BpI(AK-168) & 1 & $0 \cdot 1$ & 100 \\
Bpp(21815) & 10 & $0 \cdot 1$ & 100 \\
Bpp(AK-167) & 10 & $0 \cdot 1$ & 100 \\
Bbs(L3) & $0 \cdot 1$ & $0 \cdot 01$ & 1 \\
Bbs(H-214) & 1 & $0 \cdot 1$ & 10 \\
E. coli & 1 & $0 \cdot 1$ & 10 \\
\hline
\end{tabular}

* The minimal lethal dose (MLD) was determined by using six galactosaminesensitized mice for each dose of LPS preparation.

$\dagger$ The minimal effective dose (MED) causing pyrogenicity was determined by using three Japanese white rabbits for each dose of LPS preparation.

well as in $\mathrm{C} 3 \mathrm{H} / \mathrm{He} \mathrm{B}$ lymphocytes. We also demonstrated mitogenic activity in LPS preparations of $B$. parapertussis, $B$. bronchiseptica and B. pertussis, although at the LPS concentrations tested, only cells from $\mathrm{C} 3 \mathrm{H} / \mathrm{He}$ mice, and not $\mathrm{C} 3 \mathrm{H} / \mathrm{HeJ}$ mice, responded. The mitogenicity of two LPS preparations from $B$. parapertussis was much stronger than that of E. coli, while the LPS from Bbs(L3) showed comparable mitogenicity to LPS from E. coli. The results on mitogenic activity do not correlate with the other activities of LPS tested, which may indicate that a different mechanism is involved in the induction of mitogenicity and the other biological activities of LPS tested in this work.

The authors wish to thank Dr John J. Munoz for reviewing the manuscript.

\section{References}

amano, K., Fukushi, K. \& Watanabe, M. (1990). Biochemical and immunological comparison of lipopolysaccharides from Bordetella species. Journal of General Microbiology 136, 481-487.

ayme, G., Caroff, M., Chaby, R., Haeffner-Cavalllon, N., Dur, A. D., Moreau, M., Muset, M., Mynard, M. C., RouminantzefF, M., Schulz, D. \& Szabo, L. (1980). Biological activities of fragments derived from Bordetella pertussis endotoxin: isolation of a nontoxic, Shwartzman negative lipid A possessing high adjuvant properties. Infection and Immunity 27, 739-745.

CHABY, R. \& CAROFF, M. (1988). Lipopolysaccharides of Bordetella pertussis endotoxin. In Pathogenesis and Immunity in Pertussis, pp. 247-271. Edited by A. C. Wardlaw \& R. Parton. New York: John Wiley \& Sons.

Girard, R., Chaby, R. \& Bordenave, G. (1981). Mitogenic response of $\mathrm{C} 3 \mathrm{H} / \mathrm{HeJ}$ mouse lymphocytes to polyanionic polysaccharides obtained from Bordetella pertussis endotoxin and from other bacterial species. Infection and Immunity 31, 122-128. 
Finger, H., Heymer, B., Hof, H., Rietschel, E. T. \& SChleifer, K. H. (1976). Über Struktur und biologische Aktivität von Bordetella pertussis Endotoxin. Zentralblatt für Bakteriologie, Mikrobiologie und Hygiene (Abteilung I, Originale A) 235, 56-64.

Freinman, R., Henriksen-DeStefano, D., Tsujimoto, M. \& Vilcek, J. (1987). Tumor necrosis factor is an important mediator of tumor cell killing by human monocytes. Journal of Immunology 138, 635640.

Galanos, C., Freudenberg, M. A. \& Reutter, W. (1979). Galactosa mine-induced sensitization to the lethal effects of endotoxin. Proceedings of the National Academy of Sciences of the United States of America 76, 5939-5943.

Haeffner-Cavaillon, N., Cavaillon, J.-M. \& Szabo, L. (1982). Macrophage-dependent polyclonal activation of splenocytes by Bordetella pertussis endotoxin and its isolated polysaccharide and lipid A regions. Cellular Immunology 74, 1-13.

Haeffiner-Cavaillon, N., Cavaillon, J.-M., Moreau, M. \& Szabo, L. (1984). Interleukin-1 secretion by human monocytes stimulated by the isolated polysaccharide region of the Bordetella pertussis endotoxin. Molecular Immunology 21, 389-395.

Kumazawa, Y., Nakatsuka, H., Takimoto, H., Furuya, T., Naguro, T., Yamamoto, A., Homma, Y. J., Inada, K., Kiso, M. \& HASEGAWA, A. (1988). Importance of fatty acid substituents of chemical synthesized lipid A-subunit analysis in the expression of immunopharmacological activity. Infection and Immunity 56, 149155.

lasfargues, A., leDur, A., Charon, D., Szabo, L. \& Chaby, R.
(1986). Analysis of the lipopolysaccharide-induced cytostatic activity of macrophages, by the use of synthetic models. Cellular Immunology 98, 8-17.

Lehmann, V., Freudenberg, M. A. \& Galanos, C. (1987). Lethal toxicity of lipopolysaccharide and tumor necrosis factor in normal and D-galactosamine-treated mice. Journal of Experimental Medicine $165,657-663$.

Li, Z. M., Cowell, J. L., Brennan, M. J., Burns, D. L. \& Manclark, C. R. (1988). Agglutinating monoclonal antibodies that specifically recognize lipopolysaccharide A of Bordetella pertussis. Infection and Immunity 56, 699-702.

McGhee, J. R., Michalex, S. M., BabB, J. L., Kiyono, H., Rosenstrich, D. L. \& MERgenhagen, S. E. (1980). Lipopolysaccharide regulation of the immune response: cellular basis of adjuvanticity and suppression. In Microbiology - 1980, pp. 49-68. Edited by D. Schlessinger. Washington, DC: American Society for Microbiology.

Movat, H. Z. \& Burrowes, C. E. (1985). Local Shwartzman reaction: endotoxin-mediated inflammation and thrombohemorrhagic lesions. In Handbook of Endotoxin, vol. 3, pp. 260-302. Edited by L. J. Berry. Amsterdam: Elsevier/North-Holland Publishing Co.

NowornY, A. (1985). Antitumor effects of endotoxins. In Handbook of Endotoxin, vol. 3, pp. 389-448. Edited by L. J. Berry. Amsterdam: Elsevier/North-Holland Publishing Co.

PePpler, M. S. (1984). Two physically and serologically distinct lipopolysaccharide profiles in strains of Bordetella pertussis and their phenotype variants. Infection and Immunity 43, 224-232. 